\title{
In memoriam René Lerch
}

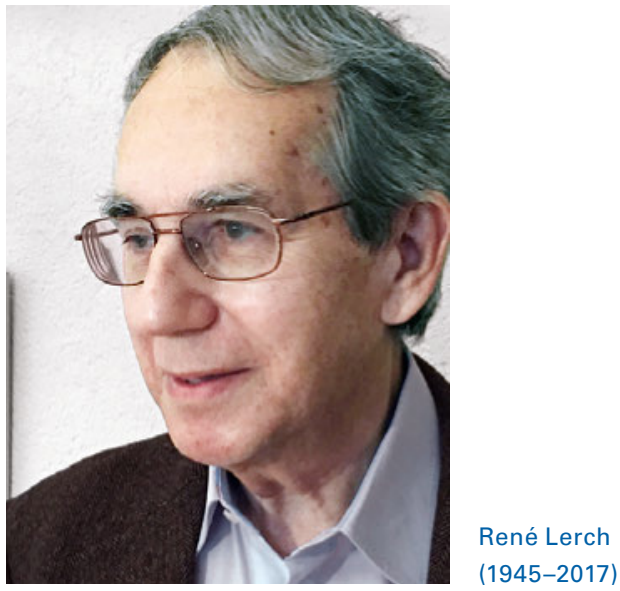

C'est avec grande tristesse et une profonde émotion, que nous avons appris le décès du Professeur René Lerch, survenu à Genève le vendredi 25 août 2017.

Né un 4 juillet 1945 à Saint-Gall, René Lerch a fait ses études de médecine à Zurich, où il obtint son diplôme en 1971.

Rapidement intéressé par la cardiologie, il se spécialise dans ce domaine sous la responsabilité du Prof. Wilhelm Rutishauser, alors chef de la cardiologie sur les bords de la Limmat.

De 1978 à 1981, René entreprend un «research fellowship» en cardiologie fondamentale à l'Université de Washington, Saint-Louis, Missouri.

De retour en Suisse en 1981, à Genève, il retrouve le Professeur Rutishauser, alors devenu chef du centre de cardiologie universitaire dans la Cité de Calvin.

\section{Pour tous ceux qui l'ont connu, et eu la chance de le côtoyer, René était un exemple.}

Chef de clinique, médecin adjoint, professeur, René à gravi tous les échelons d'une carrière académique, y compris plusieurs mois de travail comme chef de service ad intérim au début des années 2000. Pendant toutes ces années il a également réussi à diriger un laboratoire de recherche sur le métabolisme cardiaque, aboutissant à la publication de nombreux travaux scientifiques de très haute valeur.
Sur le plan national, il a été membre très actif de la Société Suisse de Cardiologie qu'il a présidée de 1998 à 2000, s'impliquant tout particulièrement dans la formation post-graduée en cardiologie (premier examen obligatoire pour l'obtention du FMH en 1999), ainsi que dans la reconnaissance des tarifs médicaux (connus actuellement sous l'appellation TARMED).

René fut également l'un des instigateurs du Cardiovascular Biology Prize, décerné chaque année par la Société Suisse de Cardiologie à un(e) jeun(e) chercheur méritant. De plus, il a été membre très actif de la Fondation Suisse de Cardiologie, dont il a présidé le comité de pilotage «insuffisance cardiaque» de 2002 à 2004. Officiellement à la retraite depuis 2010, il aura contribué pendant 20 ans, sans relâche, à la renommée clinique et scientifique de la cardiologie universitaire genevoise aux affaires de la Fondation Suisse de Cardiologie et de la Société Suisse de Cardiologie.

Pour nous, ces «internes», René était «Le» responsable de l'écho avec une expertise et une expérience difficile à égaler.

Pour tous ceux qui l'ont connu, et eu la chance de le côtoyer, René était un exemple. Les qualités qui le définissent le mieux sont certainement: intégrité, passion, précision, rigueur, engagement, disponibilité, qualités qui se font souvent trop rares de nos jours dans le monde médical. Disponible, René l'était, totalement. Qui ne se souvient d'avoir bénéficié de ses conseils avisés un samedi à $21 \mathrm{~h}$ alors qu'il était encore dans son bureau, juste pour écrire un article scientifique, parfaire des règlements de la Société Suisse de Cardiologie, ou encore corriger un projet de recherche d'un de ses collaborateurs!

Nous sommes très nombreux à avoir «quelque chose en nous de René Lerch"; ceci nous rend un peu meilleur dans notre travail quotidien. Pour tout ce qu'il nous a apporté, sans jamais compter, René restera bien ancré dans notre mémoire.

Nos pensées émues vont à sa femme Antonia, ses enfants Tobias et Serena, ainsi qu'à ses proches.

Prof. François Mach, pour le service de cardiologie des HUG Prof. Michael Zellweger, pour la Société Suisse de Cardiologie 